\title{
Laser Alarm System based on GSM Module Family
}

\author{
Liu Ying-nan, Yi Shu-juan and TaoGui-xiang \\ College of Information Technology, Heilongjiang Bayi Agricultural University, \\ Daqing 163319, China \\ Dqpilyn@126.com
}

\begin{abstract}
At present, most home alarm system mainly USES the smoke sensor and other sensors to monitor the safety of the indoor environment, and truly burglar alarm is little effect of strangers. This design is based on GSM module new home alarm system, remote control is mainly composed of AT89C52 microcontroller C programming control, through the single chip microcomputer and laser alarm interconnection communication real-time monitoring anomaly in the home, with mature and reliable GSM mobile network, real-time anomaly will be home in Chinese news sent to visually set by phone. In this way, both did to people for anti-theft and situation can be real-time reporting to his owner. Owners can also use outside messages in a timely manner to understand the home situation, circuit is simple and novel features and characteristics of the remote control, is a new type of family security alarm system.
\end{abstract}

\section{Keywords: AT89C52; The GSM module; Laser alarm}

\section{Introduction}

The family security alarm now already deeply into every household. Traditional security alarm system is mainly by the security door, window, burglar mesh, etc. Anti-theft product price does not poor, single function, but also exposed the many security hidden danger. In order to improve the traditional anti-theft devices, combined with the current science and technology, the use of electronic intelligent anti-theft system is the trend of The Times. At present, the home security alarm system on the market is not widely used in ordinary families[1]. Investigate its reason, mainly due to the performance of the anti-theft products, the price can not adapt to the requirement of the society now. So, the system in order to solve these problems, according to the specific controlled by AT89C52 microcontroller C programming the core, through single chip microcomputer and laser alarm interconnection communication real-time monitoring anomaly in the home, with mature and reliable GSM mobile network, real-time anomaly will be home in Chinese news directly sent to the mobile phone set. Make the home guard against theft system humanized operation, convenient and quick, cheap, powerful, has important practical[2].

\section{Summary of Short Message}

Short message is a mobile terminal through a mobile network to send and receive short text information communication mechanism. Text messages can be characters, Numbers, letters or symbols, and their combination. Along with the development of the GSM mobile network, users also develop to hundreds of thousands, they recognize the GSM short message service has made their life, GSM short message has become an important part of. Short message business generally to mobile users to send and receive text messages is mainly composed of digital or character, sending text messages is not more than 140 bytes. Short message store-and-forward mode is adopted, a short message sent by user after the first store in the center of the short message, and then by the short 
message center according to looking for the goal of routing information sent to the mobile terminal target mobile terminals[3]. So the GSM short message transfer is guaranteed, even if a mobile terminal temporarily inaccessible, GSM network will also keep to its message and wait for the mobile terminals appeared after the transfer in the GSM network.

\subsubsection{Short Message Technical Analysis}

AT89C52 microcontroller through the serial port directly to the TC35 module to send AT commands, can complete a variety of functions. For example, read the phone number of the SIM card, send SMS messages, receive SMS message, etc. So that it can be convenient and simple in do you want it short message transceiver, query and management. Since this design only involved in the development of short message, the following list only related to the short message of some commonly used the AT command, as shown in Table 1:

Table 1. The AT Command Associated with a Short Message

\begin{tabular}{cc}
\hline AT command & function \\
\hline AT + CMGC & Send a short message command \\
AT + CMGD & The newspaper news remove SIM card cloth \\
AT + CMGF & Choose to know news information format: 0 - PDU; 1 - text \\
AT + CMGL & List a short message in SIM card \\
AT + CMGR & Read a short message \\
AT + CMGS & Send a short message \\
AT + CMGW & To write to SIM memory short at ease \\
AT + CMSS & Send a short message from the SIM memory \\
AT + CNMI & According to the new received message \\
AT + CPMS & Select the message memory \\
AT + CSCA & Short message center address \\
AT + CSMP & Setting up the short message text mode parameters \\
\hline
\end{tabular}

\subsubsection{Short Message Mode and the Mode of Coding}

To send and receive SMS PDU mode can use three kinds of coding: 7 - bit, 8-bit and UCS2 code. 7 - bit encoding used to send ordinary ASCII characters, 8-bit codes are usually used to send data messages, UCS2 code for sending Unicode characters. Generally the PDU encoding from A B C D E F G H I J K L M thirteen.

A: length of short message center address, two hexadecimal number (1 byte).

B: short message center number type, two hexadecimal number.

$\mathrm{C}$ : the short message center number, the length of $\mathrm{B}+\mathrm{C}$ will be decided by the data in A.

D: file header byte, two hexadecimal number.

E: information types, two hexadecimal number.

F: called number length, two hexadecimal number.

G: called number type, two hexadecimal number, B value.

$\mathrm{H}$ : called number, length is decided by the data in the F. 
I: agreement, two hexadecimal number.

J: data coding scheme, two hexadecimal number.

$\mathrm{K}$ : the period of validity, two hexadecimal number.

L: length of user data, two hexadecimal number.

M: the user data, its length is determined by the data in a L. J set used UCS2 code, here is the Unicode characters in Chinese and in English.

The string to send below is "hello hello, as an example, PDU code is as follows:

0891683108409505

f004odc8685161549237f3000040112150750321oae8329bfd4697d9ec37, PDU encoding each component description as shown in table 2:

Table 2. PDU Format for each Component

\begin{tabular}{cl}
\hline subsection & \multicolumn{1}{c}{ instructions } \\
\hline 08 & The SMSC (Short Message Service Center) Short Message \\
91 & Service Center number length \\
The SMSC address types (91 international number) & Service center number (the actual number is \\
$683108409505 \mathrm{~F} 0$ & $8613800459500)$. Have a real number hi-lo exchange \\
04 & SMS to DELIVER the first eight, namely byte header files \\
0D & The length of the sending number \\
C8 & Of the number of the sending address type 15164520733 F \\
685161549237F3 & Called mobile number coding similar service center \\
00 & Protocol identifiers \\
00 & Data encoding scheme (00 said 7 bit encoding 08 said \\
40112150750321 & The code) \\
OA & The lime stamp \\
E8329BFD4697D9EC37 & User data \\
\hline
\end{tabular}

\section{The Design Requirements}

For this design, it is necessary to meet the following requirements:

1. To be able to detect the invasion of the thief alarm, and has certain concealment.

2. Able to deposit valuables user home Windows and doors and cabinets for testing, when the laser is triggered, immediately report to the police.

3. Systems that can perform sound and light alarm, and the abnormal situation in the form of text messages sent to set on the phone.

4. The user can easily control system working condition.

5. The system adopt cost-effective components as much as possible, and make the circuit design is simple and easy to understand.

\section{Design Objective}

This design is based on the GSM module family laser alarm system, complete the following main tasks:

1. Choose single chip microcomputer AT89C52, understand the basic features and functions, using ATC89C52 control system for the various functional modules. 
2. Using TC35 models of GSM short message module, monolithic integrated circuit after the acquisition to the abnormal situation, through the AT command and GSM module, serial communication, mobile phone in the form of text messages sent to the specified user exception information.

3. Use the USB download line between MCU and PC program is convenient to download.

4. Use the buzzer and light-emitting diodes (leds) as the sound and light alarm module, when the monolithic integrated circuit after the acquisition to the abnormal situation, light LED lights, and drive buzzer alarm.

5. With laser detection module, when the photosensitive resistance received laser output high level, can't get a laser, photosensitive resistance output low level, the level is the digital quantity. And then the signal changes after dealing with the transistor amplifier circuit, was sent to a single chip microcomputer, single chip microcomputer to detect the change of the level, can control the alarm sound and light alarm module. And alarm to set by a mobile phone to send text messages.

6. Design using all of the components price not too high, and in selecting device, to be in line with the principle of high cost performance.

7. The design of the hardware design part, through the simulation design is completed, and welded the real circuit.

\section{System Chart}

System block diagram is shown in Figure 1. When someone invasion, will be blocked by laser beam, photosensitive resistance to receive less than the emitted laser diode laser, will cause the change of output level, single chip microcomputer to detect the level of change will trigger the sound and light alarm module, and through the GSM module send abnormal situation to the user's phone.

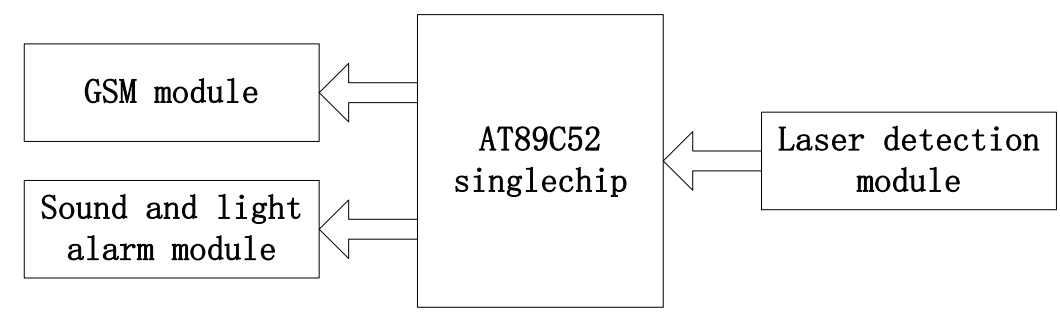

Figure 1. System Chart

\section{The Hardware System Design}

\subsubsection{Single Chip Microcomputer Minimum System Design}

AT89C52 is a low voltage, high performance CMOS 8-bit microcontroller, containing $8 \mathrm{k}$ bytes of the tablets can be repeatedly wipe Flash read-only program memory data and 256 bytes of random access memory (RAM), the device adopts high density of ATMEL company, nonvolatile storage technology production, compatible with standard MCS - 51 instruction system, built-in general 8-bit CPU and Flash memory cell, AT89C52 single chip microcomputer has been widely used in the electronics industry[4]. Single chip microcomputer minimum system is single chip microcomputer work the basic circuit, as shown in Figure 2: 


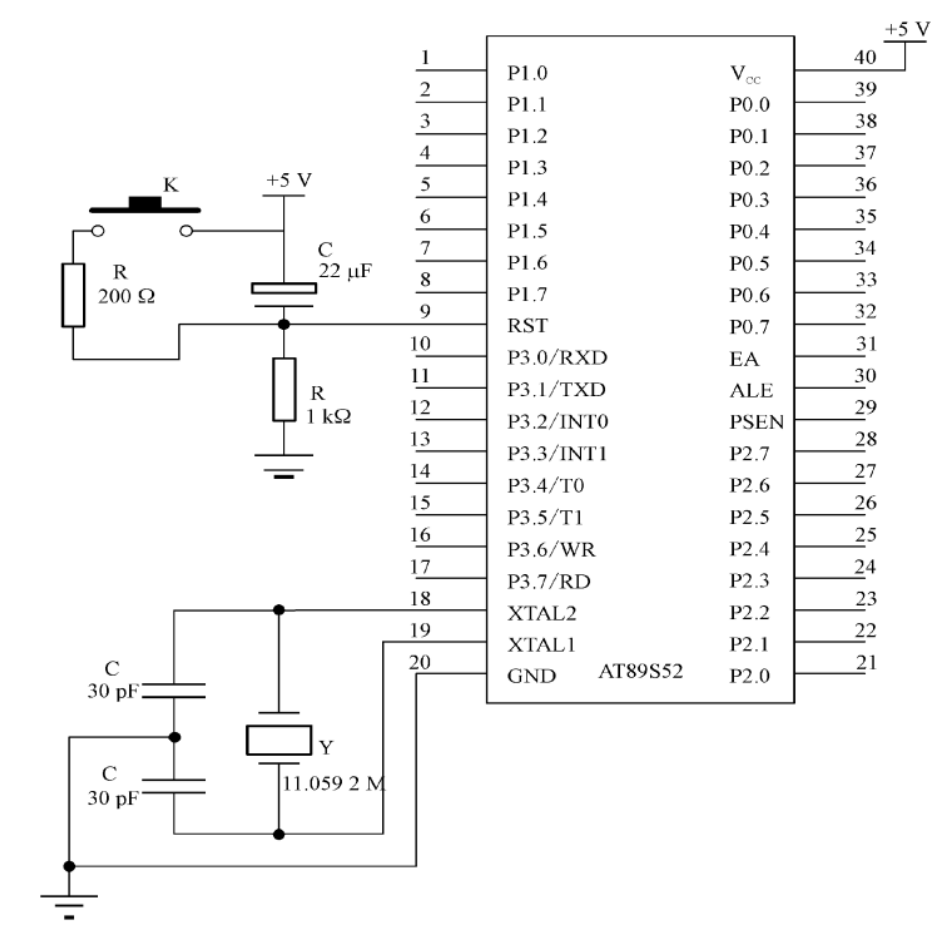

Figure 2. Single Chip Microcomputer Minimum System

\subsubsection{The GSM Module}

TC35 module is made up of power supply module (ASIC), flash memory, ZIF connectors, antenna interface of six parts. At the core of TC 35 baseband processor mainly deal with the voice and data signals inside the GSM terminal, and covers all the analog and digital function in cellular radio frequency devices. TC35 module of function block diagram is shown in Figure 3.

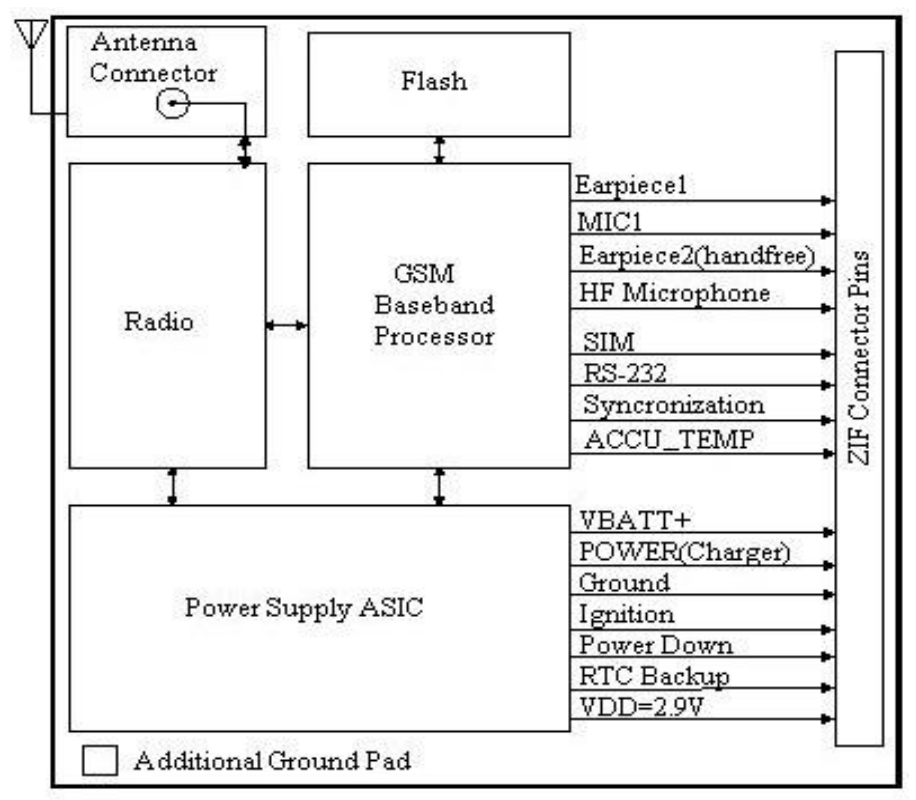

Figure 3. TC35 Function Block Diagram

TC35 module has 40 pins, through a ZIF (Zero Insertion Force, Zero resistance) socket connector lead. The 40 pin can be divided into five types, namely, power supply, 
input/output data, SIM card, audio interface and control. TC35 module pin diagram as shown in Figure 4.

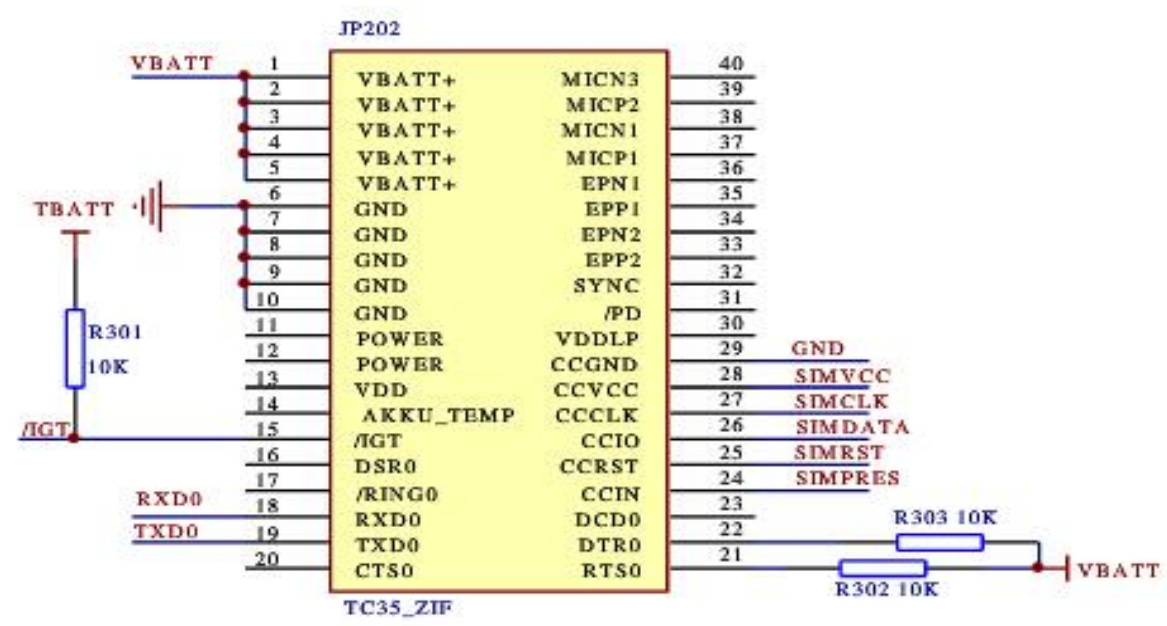

Figure 4. TC35 Pin Drawing

\subsubsection{Laser Detection Module Hardware Circuit Design}

The design of laser detection module hardware circuit as shown in Figure 5, laser used in laser diode is $4.5 \mathrm{~V}$, when the laser detection module to work normally, photosensitive resistance can receive to the laser diode emission of the laser, normal operation of the whole module[5]. When the photosensitive resistance to receive less than laser, laser detection module will trigger the sound and light alarm module.

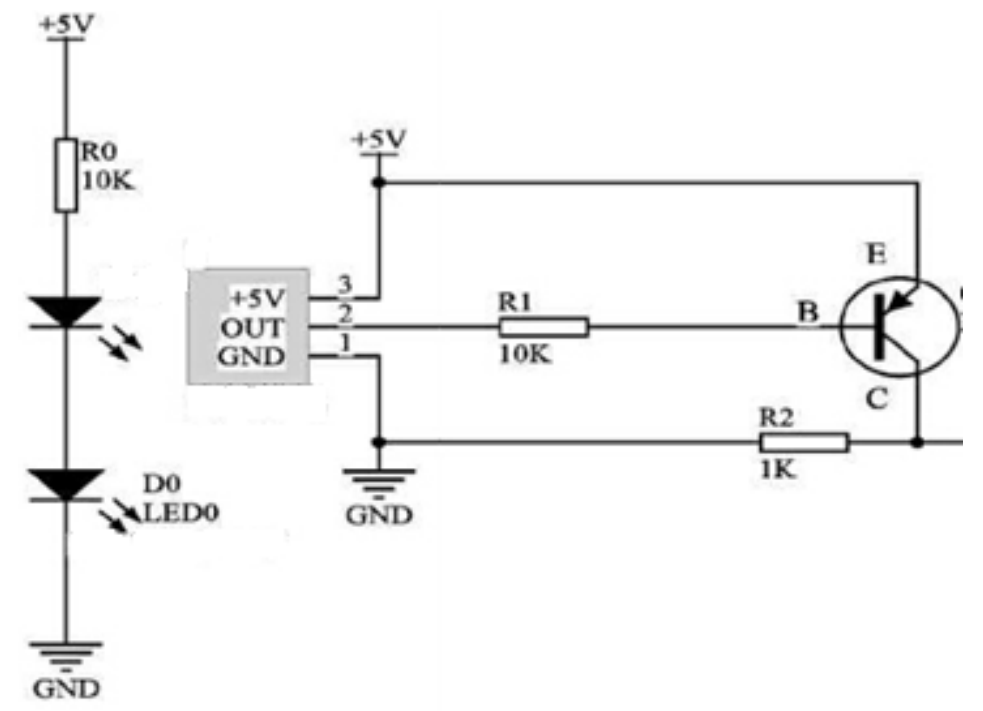

Figure 5. Laser Detection Module Hardware Circuit

\subsubsection{TC35 Hardware System Design}

TC35 module with AT89C52 single chip microcomputer communication can directly connect the serial port correspondence, at this point, connect the MCU TXD TC35 18 feet RXD, connect the MCU RXD TC35 19 feet TXD, GND connected to GND. If use $5 \mathrm{v}$ single chip microcomputer to control TC35 module, may need to be done through MAX232 level transformation[6]. Correct connection circuit of TC35 module after 
debugging. After power on, after the indicator, the TC35 has to work. When the signal lights flash, the TC35 are searching for the network, under normal circumstances within 10 seconds will slow flash ( $2 \mathrm{~s}, 1 \mathrm{~s}$ bright), then you can to a module, send and receive messages, calls etc., Function.

\subsubsection{The Working Principle of the Laser Detection Module}

Laser detection module USES a 800 - nm invisible laser technology, Easily concealed protection, generally can be installed in doors and Windows of diagonal or indoors can be installed in a horizontal or vertical. Laser detection module is by a laser beam, triode, etc. Laser is composed of $4.5 \mathrm{~V}$ of the laser diode and photosensitive resistance, can be formed between a beam or multiple lines[7-8]. Under the protection state, when the laser beam obscured, photosensitive resistance to receive less than the emitted laser diode laser, will cause the change of output level, single chip microcomputer to detect the level of change will trigger the sound and light alarm module, and the abnormal situation in the form of text messages sent to set by phone.

\subsubsection{Sound and Light Alarm Module}

Sound and light alarm module into the alarm sound and light alarm, as shown in Fig 6, the above for voice call the police, the following for light alarm, when the AT89C52 single chip microcomputer, after receipt of the abnormal signal of the detector collected for signal processing, if meet the alarm condition to trigger the alarm sound and light alarm at the same time. Buzzer work need a larger current, MCU pin can't drive, so have to drive with triode, when P2.5 output low level, triode conduction, buzzer sounding; When the P2.5 output high level, triode cut-off, buzzer don't call. Light alarm using light-emitting diodes (leds), directly controlled by single chip pins do not need additional drivers, when P2.6 output low level, light emitting diode light; When P2.6, output level led lights went out.

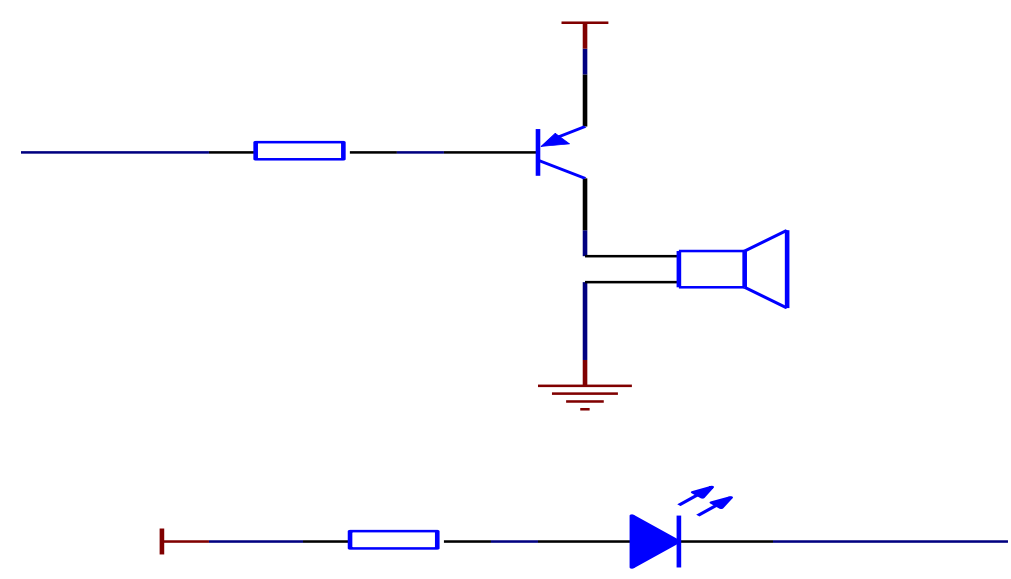

Figure 6. Acousto-optic Alarm Circuit

\section{The Software System Design}

Program for each module initialization, first of all need to be in the initialization module connected microcontroller port are defined, set the text, open the interrupt and so on. Then the single-chip computer scanning probe, abnormal signals were collected will trigger the sound and light alarm module, and mobile phone in the form of text messages sent to the specified user exception information. The main program flow as shown in Figure 7. 


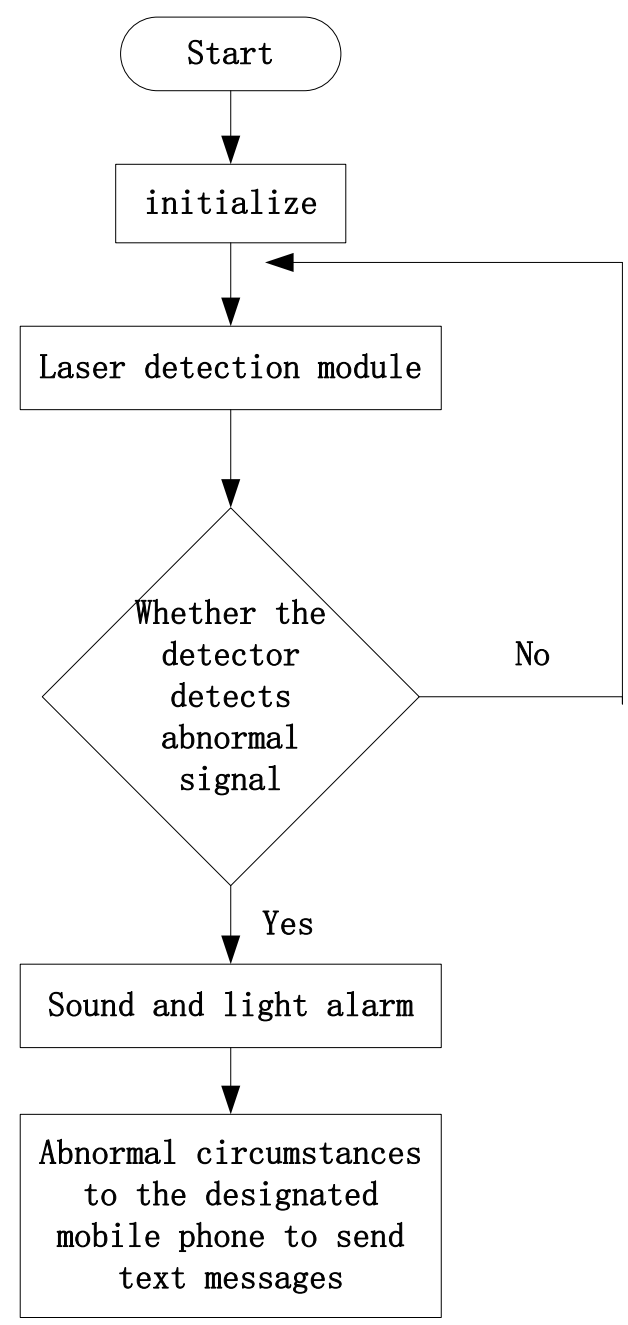

Figure 7. Main Program Flow

\section{Conclusion}

This paper designed a set of simple and efficient is suitable for modern single family home laser alarm system based on GSM module[9]. In view of the current family security alarm status both at home and abroad, and compares the existing problems in the traditional alarm system. Proposed in this paper, the design scheme. Design, USES the AT89C52 single chip microcomputer as main control chip, adopts the modular design. Through the GSM network for the development of the most stable, send alarm messages to the user, with high efficiency, the advantages of stability, has a high use value. Using the innovative laser detection module, application of sound and light alarm buzzer and light-emitting diodes. Finally completed the rigid circuit board debugging and display. The effect of income in accordance with the desired effect.

\section{Acknowledgments}

1. State "thirteenth five-year" The national key research opportunity corpus (2016YFD0701801).

2. State "thirteenth five-year" Research and development of the national key program corpus (2016YFD0701801-03). 


\section{References}

[1] Chen Ningpo, cai-hong feng, ling-yun CAI, zhen-zhou wang, leudon. Family security alarm system based on GSM short message design [J]. Journal of hebei industrial science and technology, 2013, 30 (02) : 104-108.

[2] Li Jun, Li Murong, Xin Jianju, Lai Beixuan, Ma Qiuci. Wireless Sensor Network for Indoor Air Quality Monitoring [J]. Journal of Sensors and Transducers, 2014, 172 (6) : 86-90.

[3] Tony. Based on the GSM module and the ARM of the smart home environment safety monitoring system design [J]. Journal of jilin engineering technical teachers college, 2013, 29 (7) : 76-79.

[4] Huang Shixuan, zheng Eric yi, li ping. Intelligent security alarm system based on GSM module design [J]. China's new communications, 2013, (15) : 90-91.

[5] Wei Yuyan. Introduction to the GSM module used in family security alarm system of teaching design [J]. Journal of professional, 2010 (9) : 74-76.

[6] Huang Xinrong. Family security alarm system based on GSM SMS module design [J]. China's new communications, 2010, 12 (9) : 83-85.

[7] by family security alarm system based on GSM SMS module [J]. Journal of shaoxing liberal art \&science college (natural science), 2010, 30 (4) : 69-72.

[8] Cai Xiaoyan, xifang feiyingli zuzhi lilun shuping [Wang Zhaoping. Based on GSM SMS module family anti-theft alarm circuit design [J]. Journal of experimental science and technology, 2015, 13 (3) : 20 to 22.

[9] Chen Yan pairs, jia-hong Yang. Home wireless alarm system based on GSM SMS module [J]. Computer knowledge and technology, 2015, 11 (18) : 183-184 + 187.

[10] Han - Chiang Chen, Y., m. Huang, Chia - Hung, Su, Te - Wei Chiu. The Implementation of SOPC -based Telecom and Datacom for monitoring wireless sensor networks [J]. Journal of Telecommunication Systems, 2013, 52 (4) : 2325-2333.

\section{Authors}

\section{Liu Ying-nan}

Author's profile: Liu Ying-nan(1982-), Heilongjiang Province, Anda. Lecturer. Master. Email:dqpilyn@126.com.Telphone:15845962618

\section{Yi Shu-juan}

Correspondent author: Yi Shu-juan(1965-),Shandong Province, Qixia. Professor. doctoral supervisor. 
International Journal of Smart Home

Vol. 11, No. 5 (2017) 